\title{
Not one odour but two: A new model for nestmate recognition
}

\author{
Philip Newey* \\ Department of Ecology and Evolution, Biophore, University of Lausanne, 1015 Lausanne, Switzerland
}

\section{A R T I C L E I N F O}

\section{Article history:}

Received 15 June 2010

Received in revised form

28 August 2010

Accepted 25 October 2010

Available online 30 October 2010

Keywords:

Recognition systems

Nestmate recognition

Colony odour

Aggression

\begin{abstract}
A B S T R A C T
Recognition systems play a key role in a range of biological processes, including mate choice, immune defence and altruistic behaviour. Social insects provide an excellent model for studying recognition systems because workers need to discriminate between nestmates and non-nestmates, enabling them to direct altruistic behaviour towards closer kin and to repel potential invaders. However, the level of aggression directed towards conspecific intruders can vary enormously, even among workers within the same colony. This is usually attributed to differences in the aggression thresholds of individuals or to workers having different roles within the colony. Recent evidence from the weaver ant Oecophylla smaragdina suggests that this does not tell the whole story. Here I propose a new model for nestmate recognition based on a vector template derived from both the individual's innate odour and the shared colony odour. This model accounts for the recent findings concerning weaver ants, and also provides an alternative explanation for why the level of aggression expressed by a colony decreases as the diversity within the colony increases, even when odour is well-mixed. The model makes additional predictions that are easily tested, and represents a significant advance in our conceptualisation of recognition systems.
\end{abstract} (c) 2010 Elsevier Ltd. All rights reserved.

\section{Introduction}

Recognition systems play a key role in a range of biological processes, including mate choice (Pusey and Wolf, 1996), immune defence (Hornung and Latz, 2010) and the evolution of altruistic behaviour (Hamilton, 1964; Mateo, 2004). For animals that live in groups it is particularly important to be able to recognise which individuals belong to one's own group and which do not, in order to protect resources and brood and to maintain colony integrity. This may be quite easy when groups are small and when individuals have well-developed cognitive abilities. However, in very large groups, particularly when cognitive abilities are limited by small brain size, differentiating between group members and others becomes more difficult. Social insects provide valuable models for exploring how recognition systems operate under these difficult conditions.

Although social insects generally discriminate between nestmates and non-nestmates, the system appears to be far from perfect, and the aggression directed towards intruders by workers can vary enormously even within a colony. Type II recognition errors (Reeve, 1989), where workers mistake alien conspecifics for nestmates, appear quite common, although Type I errors (Reeve, 1989), where workers mistake nestmates for alien intruders, appear extremely rare. If workers use the same template, such as the shared colony odour (Crozier and Dix, 1979; Crozier and

\footnotetext{
* Tel.: +4121692 4193; fax: +41216924165

E-mail address: philip.newey@unil.ch
}

Pamilo, 1996), with which to assess intruders, this variability must be attributed to workers having different thresholds for aggression (Reeve, 1989) or undertaking different roles within the colony (Hölldobler, 1983). Thresholds may vary according to context (Reeve, 1989), be modified by prior experience (Van Wilgenburg et al., 2010), or change with age (Hölldobler, 1983). Nevertheless, other things being equal, the threshold model predicts that under given conditions individual $A$ will always be more aggressive than individual $B$ if the former has a lower acceptance threshold. Recent empirical evidence from the weaver ant Oecophylla smaragdina suggests that this is not always the case. Newey et al. (2010) first showed that variation in aggression between workers in a recipient colony and conspecific intruders was due more to differences between the aggressors than their victims. They then demonstrated that a recipient could be more aggressive towards intruders from one colony than its nestmate, but less aggressive towards intruders from a different colony (Newey et al., 2010). In all cases, as far as practicable, the context was unchanged. Existing models cannot account for this type of interaction.

In a colony in which odour cues are shared among workers, each worker may be described in terms of both an innate odour and an expressed odour. The innate odour is that which the individual would express if kept in isolation from the colony, and which presumably has a genetic basis (Boulay et al., 2000). The expressed odour is the odour that results from the mixing of cues among workers via trophollaxis and allo-grooming and, depending upon the efficiency with which odour is shared, will be similar for all workers. This odour may also be influenced by environmental factors such as diet and nest substrate (Buczkowski et al., 2005; 
D'Ettorre et al., 2006) and season (Katzerke et al., 2006). For the purposes of the present model I am assuming that odour is principally of genetic origin, although including an environmental component does not alter the fact that there are essentially two odours, the individual's innate odour and the shared colony odour. It is this expressed odour rather than the individual's innate odour that is detected by extracting cuticular hydrocarbons. Here I propose a new model for nestmate recognition based on both of these odours, rather than just the shared colony odour. This new model complements existing models and accounts for the recent findings concerning weaver ants. It retains the concepts of gestalt odour and thresholds, but presents them in a novel way that explains a broader range of behaviour than earlier models. It also provides an alternative explanation for why aggression may be reduced in colonies with high genetic diversity, even when odour is well-mixed (Martin et al., 2009). Finally, the model makes additional predictions that are easily tested.

\section{Materials and methods}

\subsection{Model development}

Consider an $n$-dimensional odour space in which the position of the individual innate odour and the shared colony odour are located. This multi-dimensional space would be similar to that generated by a principal components analysis of the cuticular hydrocarbon profiles of individuals and colonies. The fact that ants often require several minutes and many interactions before committing themselves to an act of aggression suggests that they weigh several factors before making a decision, which is consistent with the multi-dimensional odour space proposed here.

Let the innate odour of worker $W_{i}$ be $\omega_{i}$, and the shared colony odour be $\Omega$. The relationship between these odours may be represented by a vector $\overrightarrow{\omega_{i} \Omega}$ (Fig. 1), the vector template. A zone of tolerance is defined for each worker, described by an ellipse (in two dimensions) or ellipsoid (in $n$-dimensions) with $\omega_{i}$ and $\Omega$ as the foci, and with $\Omega$ arbitrarily located at the origin. An ellipsoid form is

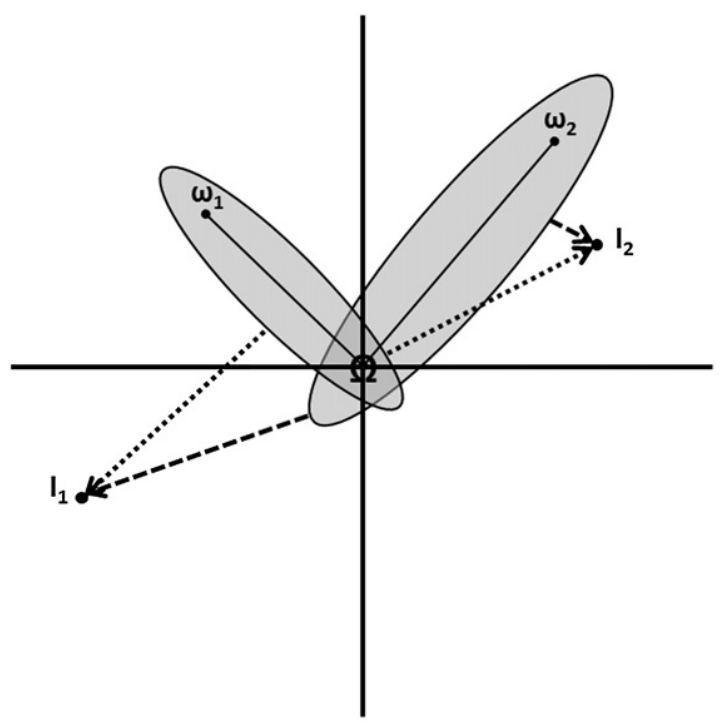

Fig. 1. Two dimensional illustration of the vector template model. Two recipients from a colony with shared expressed odour $\Omega$ but with innate odours $\omega_{1}$ and $\omega_{2}$, respectively, are depicted in the two-dimensional odour space. The ellipse around each vector represents the zone of tolerance for each worker: intruders falling within that zone are treated as nestmates. $I_{1}$ and $I_{2}$ represent intruders from two other colonies within the population. The level of aggression shown by each recipient towards each intruder is proportional to the dashed lines from the edges of the ellipses to the positions of the intruders. adopted for this zone of tolerance because it makes intuitive sense that the zone of tolerance will be narrower around the worker's own innate odour and the shared odour of the colony. This form also has mathematical properties that simplify the calculations. However, other forms could be used for this zone of tolerance without qualitatively changing the results.

Any intruder falling within this zone of tolerance is treated as a nestmate. It is assumed that as $\overrightarrow{\omega_{i} \Omega}$ increases in magnitude, the proportions of the ellipse (that is, the ratio of the long axis to the short axis) remain constant (Fig. 1). The zones of tolerance of different workers overlap around $\Omega$, and assuming that odour is well-mixed within the colony, the expressed odour of all workers will fall close to $\Omega$, avoiding aggression between nestmates. This does not depend on the level of genetic variation within the colony, but only on how effectively odour is shared. If odours are not wellmixed within the colony then there is no colony gestalt odour and models involving individual recognition will be more appropriate (Crozier and Dix, 1979).

Consider two workers $W_{1}$ and $W_{2}$ from the same colony with innate odour $\omega_{1}$ and $\omega_{2}$, respectively (Fig. 1). Let their respective vector templates be $\overrightarrow{\omega_{1} \Omega}$ and $\overrightarrow{\omega_{2} \Omega}$. Each worker will respond nonaggressively to intruders whose odour falls within their zone of tolerance. The level of aggression exhibited towards intruders outside these zones will be proportional to the distance outside the zone. Thus, although there is a zone in which aggression does not occur and a zone in which it does, there is not an abrupt transition from non-aggression to maximum aggression, as threshold models seem to imply. Rather, aggression gradually increases as the distance outside the zone of tolerance increases. This seems more in accordance with empirical findings in which aggression is generally scored on a continuous scale (Roulston et al., 2003). Because individuals within a particular colony have zones of tolerance with different volumes, the model includes the possibility that workers have different overall thresholds for aggression.

The properties of an ellipse or ellipsoid are such that the sum of the distance of each focal point, $a$ and $b$, to a given point $p$ on the surface of the ellipsoid is always equal to the length of the long axis $L: \overline{a p}+\overline{b p}=L$. Therefore, any point $p$ such that $\overline{a p}+\overline{b p} \leq L$ falls within the ellipse, while any point $p$ such that $\overline{a p}+\overline{b p}>L$ falls outside the ellipse. If $\overline{a p}+\overline{b p}=L$ then the level of aggression $A$ is determined by $A=\beta(L-L)$ for $L>L$, where $\beta$ is a simple scaling factor. I assume that this is a linear relationship, but any monotonically increasing function would serve the same purpose. If two intruders $I_{1}$ and $I_{2}$ (Fig. 1) are encountered from colonies located in different positions within the odour space, $W_{1}$ and $W_{2}$ are expected to have different responses to these intruders.

\subsection{Demonstrating the model}

A three-dimensional version of the model was used for generating its predictions, but this can easily be generalised to higher dimensions, and a modified one-dimensional version is also valid. Three dimensions represent a compromise which restricts computation to a reasonable level. The intention here is to present a qualitative rather than a precise quantitative demonstration of the model.

Recipients were randomly selected from a colony with mean innate odour $\Omega=[0,0,0]$ and standard deviation $(\mathrm{SD})=1$, unless otherwise indicated. Note that this is the SD of the innate odours of the individuals making up the colony, not the SD of the expressed odour of individuals within the colony. A random point $P=[x, y, z]$ was chosen from a normal distribution with mean $=[0,0,0]$ and $\mathrm{SD}=5$. This point was then used to represent the mean expressed odour across the entire population, and determined the position of the recipient colony's odour, which remained fixed at $\Omega=[0,0,0]$, 
with respect to the distribution of odour within the population. Varying the position of the population mean rather than the recipient colony mean simplified calculations. Intruder colonies were then selected at $P_{i}=\left[x_{i}, y_{i}, z_{i}\right]$ from a distribution with mean $P$ and $\mathrm{SD}=5$. These colonies were thus chosen at random with respect to the mean population odour. Finally, individual intruders were selected from these colonies assuming a mean odour $P_{i}$ and $\mathrm{SD}=0.2$, unless otherwise indicated. The SD referred to here is that of the expressed odours of individuals within the intruder colony, not that of their innate odours. This was set lower than the SD of the innate odours of workers in the recipient colonies because innate odours are mixed, reducing the variability in expressed odour. Thus, in the case of the recipients, SD is that of the workers' innate odours, while in the case of the intruders, SD is that of the workers' expressed odours, which will always be lower if odour is shared.

Ten simulations were conducted to test for each of the following.

\subsubsection{Interactions between recipients}

In each simulation, two recipients were confronted with 10 intruders from each of two intruder colonies. Aggression was compared using repeated measures ANOVA.

\subsubsection{Source of variation}

Twenty recipients were selected and confronted with a single intruder. Then 20 intruders from a single colony were selected and presented to a single recipient. This was repeated for intruder colonies with SD ranging from 0.2 to 1.0 in steps of 0.2 , representing a gradual decrease in the efficiency of odour-sharing. The mean variance with different recipients was compared to the mean variance with different intruders for each value of SD using repeated measures ANOVA.

\subsubsection{Effects of increased diversity}

A colony with greater genetic diversity presumably has greater diversity in $\omega_{i}$ than one with low genetic diversity. The effect of this on both the mean level of aggression and variability in aggression was determined when the recipient colony encountered (i) several intruders from the same colony and (ii) several intruders from different colonies. Twenty recipients were selected from a colony with SD ranging from 0.2 to 1.0 in steps of 0.2 , representing increasing genetic diversity, and confronted with 20 intruders from a single colony $(S D=0.2)$. Then 20 recipients from a colony with $S D$ ranging from 0.2 to 1.4 in steps of 0.2 were confronted with 20 intruders from anywhere within the population $(S D=5)$. A greater range of SD for the recipient colony was used in the second part of the experiment because the effect was less pronounced (see Results). The results were analysed using linear regression models with SD of the recipient colony as the predictor variable and either mean or SD of aggression as the response variable.

Visual C+ 2008 Express Edition (2)2007 Microsoft Corporation was used for carrying out the simulations. Statistical tests were conducted with PASW Statistics 18 @2009 SPSS Inc. Tests were two-tailed with $\alpha=0.05$

\section{Results}

The mean level of aggression expressed towards alien conspecifics randomly selected from colonies within this hypothetical population was $2.40 \pm 1.06$ (mean \pm standard deviation), while that expressed towards nestmates was only $0.18 \pm 0.09$, which was significantly lower (Welch's $t$-test: $t_{19.25}=9.36, p<0.001$ ). The variance of the latter was also significantly less than that of the former (Levene's test: $F_{1,38}=20.31, p<0.001$ ).

\subsection{Interactions between recipients}

In simulations with randomly chosen recipients and intruders, in 8 out of 10 cases there was a significant interaction between the responses of recipients to intruders from different colonies (in all simulations except 3 and 9, $p<0.001$; Fig. 2). In simulations 3 and 9 , one recipient was consistently more aggressive than the other $(p<0.001$ in both cases $)$.

\subsection{Source of variation}

The variation expressed towards intruders could be attributed more to differences between aggressors than to differences between their targets. When the standard deviation in expressed odour was less than that of innate odour (that is, when there was a significant degree of odour-sharing among workers) variation in aggression was significantly greater when different recipients faced the same intruder than when a single recipient faced several intruders from the same colony (Fig. 3). When SD of expressed odour of the intruder colony was equal to that of innate odour of the recipient colony, there was no difference in the contribution made by recipients and intruders to variation in aggression. a

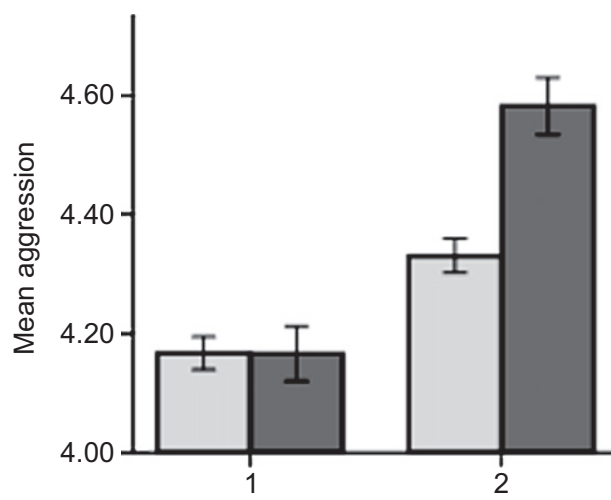

b

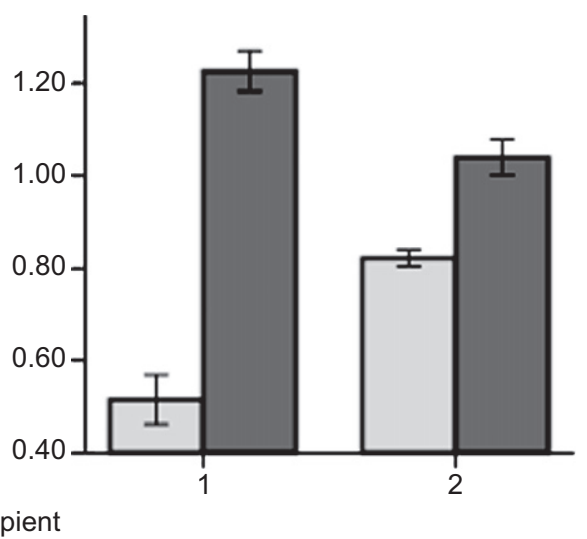

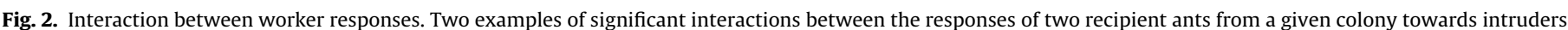

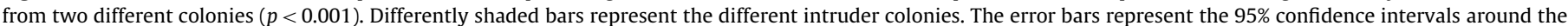

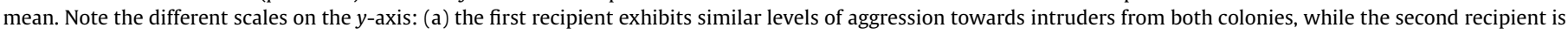

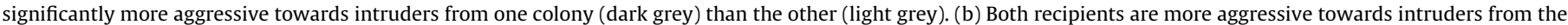
dark grey colony than the light grey colony, but the difference is much greater for the first recipient. Interactions occurred in 8 out of 10 simulations. 


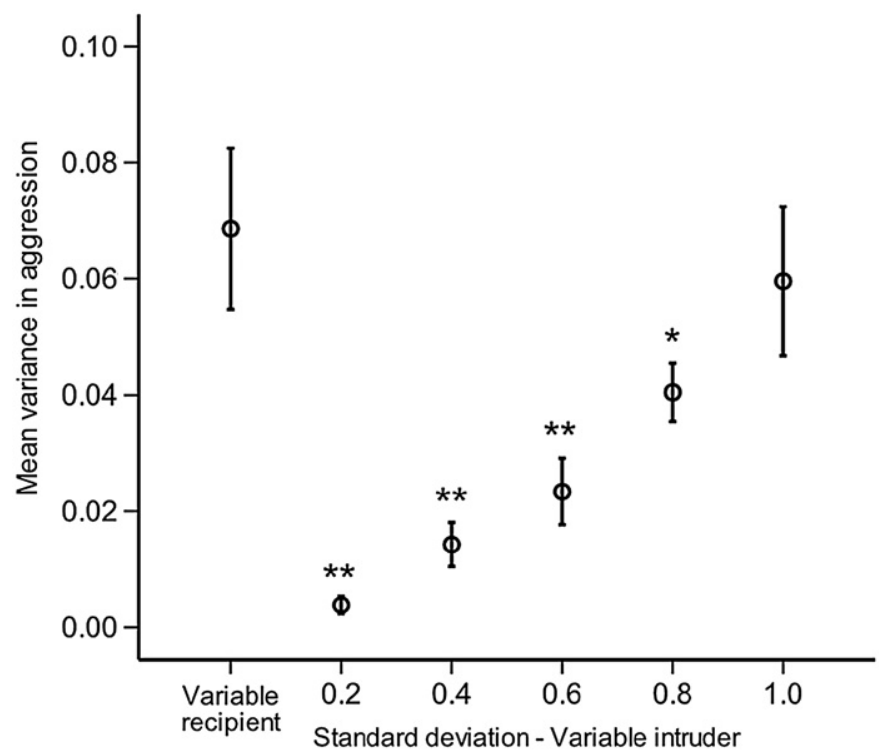

Fig. 3. Source of variability in aggression. The mean variance of aggression when the identity of the recipient varied (leftmost point) was compared with the mean variance of aggression when the identity of the intruder varied, with different levels of variability (standard deviation) in the shared odour of the intruder colony. The error bars represent the $95 \%$ confidence intervals around the mean. A significant difference between the variance when the identity of the recipient varied and when the identity of the intruder was varied is indicated by *at the 0.01 level and **at the 0.001 level.

\subsection{Effects of increased diversity}

Mean aggression expressed towards intruders from a single colony decreased significantly as variability in $\omega_{i}$ increased (Fig. 4a: $\left.F_{1,48}=74.07, R^{2}=0.607, p<0.001\right)$. It also decreased significantly when intruders were derived from a variety of colonies, although the effect was less pronounced $\left(F_{1,78}=7.341, R^{2}=0.086, p=0.008\right)$. Variability in aggression increased when intruders were derived from a single colony (Fig. $4 \mathrm{~b}: F_{1,48}=130.91, R^{2}=0.732, p<0.001$ ), but not when they were derived from several colonies $\left(F_{1,78}=0.938, R^{2}=0.012, p=0.336\right)$. This is because variation among the intruders was much greater when they were selected from any colony within the population rather than from a single colony.

The decrease in mean aggression expressed towards intruders from a single colony combined with an increase in the variability of the response indicates that the decrease in mean aggression did not occur simply because all individuals within the colony became less aggressive. Rather, there was an increase in both low level and high level responses at the cost of moderate responses, although low level responses showed a greater increase, reducing the overall mean (Fig. 5). To understand this, consider a simple example in which $\Omega, \omega_{i}$ and the position of an intruder are collinear. As $\omega_{i}$ moves closer to the position of the intruder, aggression decreases until it reaches zero when the intruder falls within the recipient's zone of tolerance. It remains zero no matter how much further $\omega_{i}$ proceeds in that direction along the line. Now consider what happens as $\omega_{i}$ moves away from the position of the intruder and closer to $\Omega$ : aggression increases until it reaches a maximum when $\omega_{i}=\Omega$. However, if $\omega_{i}$ continues to move away from the position of the intruder, aggression will again begin to decrease as the zone of tolerance grows. Thus, as the variability in $\omega_{i}$ increases, the probability of both high and low level aggressive responses increases, but the probability of a low level response increases at a greater rate. a

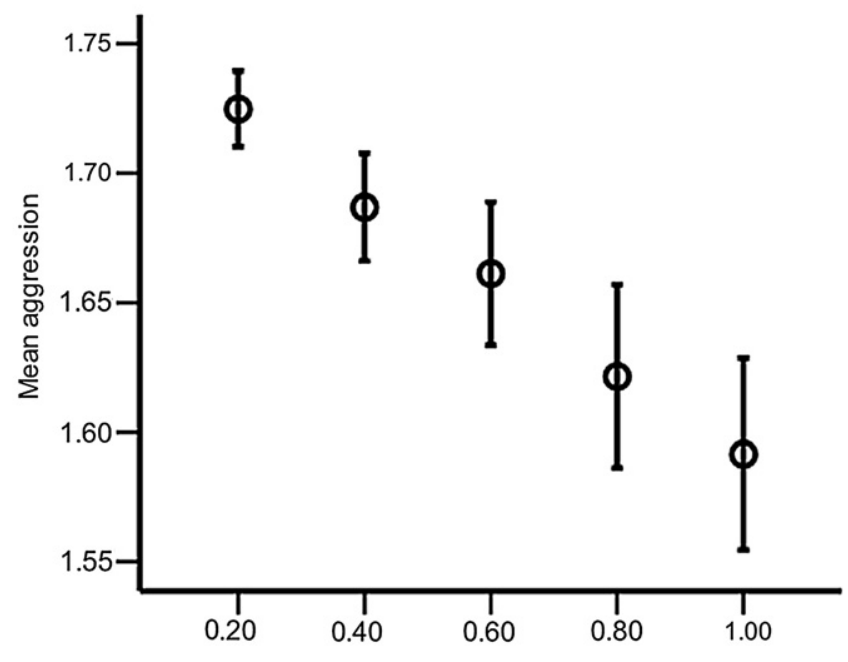

b

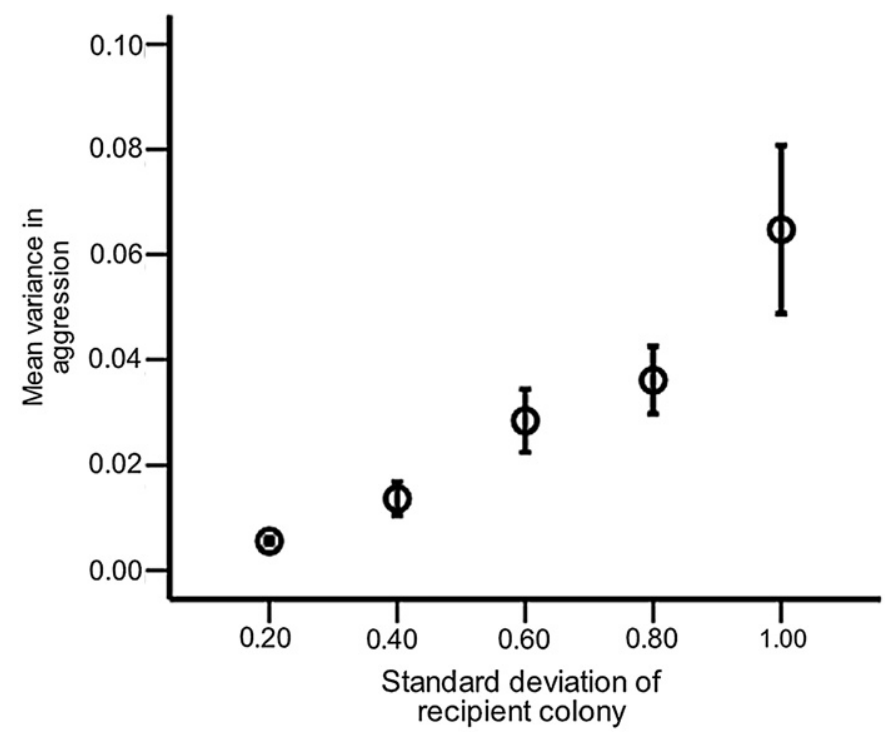

Fig. 4. Change in mean and variance of aggression with increasing diversity in the recipient colony. The mean level of aggression (a) expressed towards intruders from a single colony decreased significantly as the variability in the innate odours of individuals within the recipient colony increased, while the mean variance (b) increased. The error bars represent the $95 \%$ confidence intervals around the mean

\section{Discussion}

This model provides a new mechanism for understanding both individual and colony level differences in aggression. It offers a new way of viewing template formation, involving both the individual's innate odour and the shared colony odour. It explains why individuals have zones of tolerance, in which they are prone to accept non-nestmates as nestmates, but rarely the other way around. The low level of aggression expressed towards nestmates $(0.18 \pm 0.09)$ is unlikely to lead to any significant conflict within the colony, supporting the general observation that Type I recognition errors are rare.

The model also provides a clear account of the interaction observed in the responses of different weaver ant workers towards different intruders (Newey et al., 2010). Different workers within the recipient colony respond in different ways to intruders from various alien colonies. Further work on additional species should be 


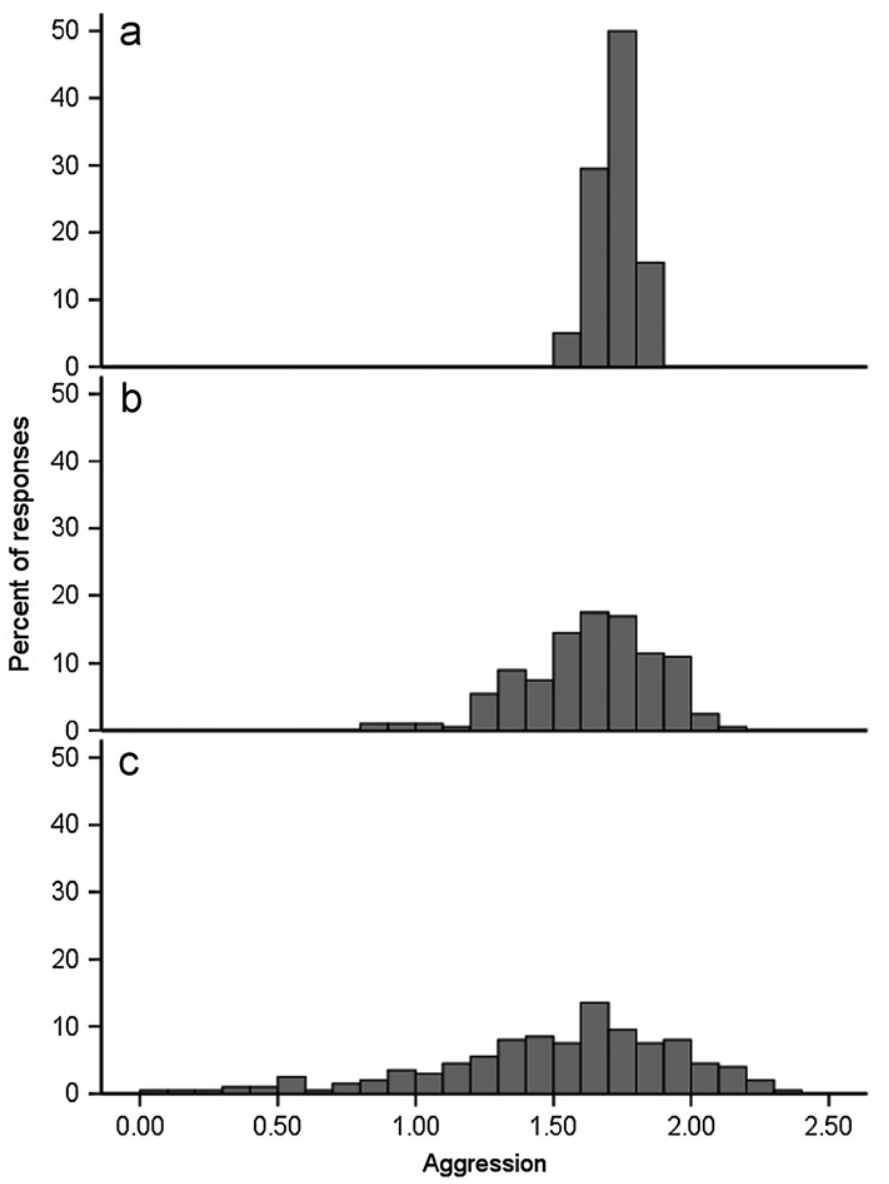

Fig. 5. Frequency distribution of aggressive responses. The distribution of aggressive responses changed as the standard deviation in the innate odours of workers in the recipient colony increased. The distribution is shown for standard deviation (a) 0.2 , (b) 1.0 and (c) 2.0 .

conducted to see how widespread this phenomenon may be. The model does not preclude the possibility that some workers may be generally less tolerant than others (having a smaller zone of tolerance or lower response thresholds), that this may vary depending on the context or on physiological factors, or that some workers may be more actively involved in a defensive role. However, it adds a new component that accounts for much of the unexplained variation that is frequently observed among workers. From a biological perspective it is not unreasonable to suppose that a worker has an awareness of both its own innate odour and the shared colony odour, and that both of these play a role in determining its relationship to other individuals that it encounters. The model does not assume (or preclude) the existence of a neural template for the shared colony odour. A worker may acquire knowledge of this odour either via contact with nestmates, or directly through sampling its own expressed odour. This avoids the need to constantly update the template as colony odour changes over time. As an individual presumably continues to generate those chemicals that contribute to its particular innate odour it is reasonable to assume that it has direct knowledge of this odour, which may also change as, for example, the individual ages or changes its role within the colony.

When colony odour is well-mixed, the model also explains why variability in aggression arises more from variation in recipients than intruders, as found for O. smaragdina and also Rhytidoponera confusa (Crosland, 1990), the only two species in which this has been tested to date. This is only the case when the variability of the innate odours of recipients exceeds that of the expressed odours of intruders. When the variability is the same, differences among intruders and recipients contribute equally to variation in aggression. However, this represents a situation in which odour cues are not shared among workers, in which case it makes little sense to speak of a gestalt colony odour, and an alternative recognition system would need to be in place.

Like other models, and as observed in several ant species (Pirk et al., 2001; Starks et al., 1998), the model predicts that the greater the diversity of innate odours (genetic diversity) in the recipient colony, the lower the mean level of aggression expressed. Breed and Bennett (1987) proposed that this reduction in aggression occurs because in a colony with high genetic diversity the mixing of individual odours results in a shared odour that is closer to the overall population mean. However, this model cannot account for the interactions among workers described above; nor can it explain why the variability in the response is mostly due to recipient ants rather than intruders. Others have suggested that this reduced level of aggression results from an increase in the diversity of recognition cues among workers (Martin et al., 2009; Starks et al., 1998), which presupposes an inefficient mixing of cues. However, it is not clear why this should lead to a reduction in the mean aggression rather than to an increase in the variance of aggression: some ants might become more aggressive, while others become less aggressive, but the mean remains the same. The present model effectively combines these two perspectives: the diversity of recognition cues increases within colonies with greater genetic diversity, regardless of whether colony cues are well-mixed (Martin et al., 2009). This genetic variation continues to play a role in the recognition system via the innate odours of individuals. The present model predicts both an increase in the variance and a decrease in the mean of aggression with greater genetic diversity. It further predicts that the reduction in mean aggression when diversity increases arises because the frequency of low aggressive responses increases at a greater rate than the frequency of high aggressive responses (although both increase), rather than because all ants within the colony become less aggressive, as predicted by the model of Breed and Bennett. This has never been tested, largely because this variation is obscured by reports of mean colony values. Finding support for this prediction would go a long way towards validating the present model.

What would favour the evolution of a recognition system that is influenced by both the individual's innate odour and the shared colony odour? In colonies with low genetic diversity, it may be possible for a zone of tolerance to encompass the few odour phenotypes that exist within the colony, without greatly increasing the probability of mistaking a non-nestmate for a nestmate (Type II error). As colonies increase in genetic diversity, it becomes more difficult to include additional phenotypes within this zone of tolerance without also increasing Type II errors. The sharing of recognition cues among workers again reduces the number of phenotypes within the colony, potentially to a single phenotype. However, as the individual continues to generate the chemical cues that constitute its innate odour, without constant mixing the individual's expressed odour will be continuously drawn away from the gestalt odour back towards its innate odour, somewhere along the vector $\overrightarrow{\omega_{i} \Omega}$. Empirical support for this is found in a study of Camponotus fellah, with workers expressing an individual genetically determined hydrocarbon profile after a period of isolation from the colony (Boulay et al., 2000). Stochastic fluctuations in the efficiency with which odour cues are exchanged within the colony may maintain a dynamic tension between the individual innate odour and the shared colony odour. In these circumstances, the most parsimonious solution may be to maintain a twophenotype system such as that described here, which results in 
the inclusion of phenotypes along and around $\overrightarrow{\omega_{i} \Omega}$ within the zone of tolerance.

Including the individual's own innate odour in the recognition process ensures that there will be a variety of templates used by workers to identify intruders: different workers will make Type II errors with respect to different intruders, but there are likely to be some workers that correctly identify each intruder. In this way Type I errors are avoided without dramatically increasing the number of Type II errors. The fact that colony odour often changes over time (Newey et al., 2009; van Zweden et al., 2009; Vander Meer et al., 1989) may further maintain uncertainty around the shared colony odour, preventing selection against this bipolar recognition system, even when odour-sharing is efficient.

The model presented here is prompted by a small number of studies, but the phenomena described in those studies are likely to be much more widespread. The lack of studies in this area reflects a tendency to deal with aggression at the colony level rather than the individual level, with variation in the latter being regarded as random noise. Focusing on the individual reveals that there is structure in this variation which can help to shed light on the process and evolution of nestmate recognition. This relatively simple model has greater explanatory power than previous models, providing a more thorough account of individual variation among workers, and providing an alternative mechanism for understanding why high diversity colonies may display lower levels of aggression than low diversity colonies, even when odour is wellmixed. It explains why variation in aggression depends more on the aggressor than the victim. It also accounts for the rarity of Type I recognition errors compared to Type II errors, but with the important proviso that when one worker makes a Type II recognition error viv-a-vis a given intruder, there are likely to be other individuals within the nest that make a correct identification. This would not be the case if all individuals used the same recognition template. The presence of different thresholds within the colony could correct for this, but those individuals with lower thresholds would also tend to be more aggressive towards nestmates whose odours diverged somewhat from the shared odour. The present model provides for the operation of diverse recognition systems within the colony without increasing the risk of intra-nest aggression. Finally, the model makes additional predictions that are easily tested, and which should encourage more research into individual variation in aggression, an area almost completely neglected up to the present time.

The more we understand about recognition among social insects, which appears to be particularly demanding in large colonies, the constituent individuals of which have limited cognitive abilities, the more our understanding of recognition systems in general will be advanced. I suggest that this understanding will be even further advanced if we begin to pay closer attention to the variation among individuals, which may contain valuable information which is obscured when only mean colony values are considered.

\section{Acknowledgements}

I thank L. Keller for comments on the MS and N. Perrin for suggestions concerning the model. P.N. was supported in this project by a grant from the Swiss NSF to Laurent Keller.

\section{References}

Boulay, R., Hefetz, A., Soroker, V., Lenoir, A., 2000. Camponotus fellah colony integration: worker individuality necessitates frequent hydrocarbon exchanges. Anim. Behav. 59, 1127-1133. doi:10.1006/anbe.2000.1408.

Breed, M.D., Bennett, B., 1987. Kin recognition in highly eusocial insects. In: Fletcher, D.J.C., Michener, C.D. (Eds.), Kin Recognition in Animals. John Wiley \& Sons, Chichester.

Buczkowski, G., Kumar, R., Suib, S.L., Silverman, J., 2005. Diet-related modification of cuticular hydrocarbon profiles of the Argentine ant, Linepithema humile, diminishes intercolony aggression. J. Chem. Ecol. 31, 829-843. doi:10.1007/s10886-005-3547-7.

Crosland, M.W.J., 1990. Variation in ant aggression and kin discrimination ability within and between colonies. J. Insect Behav. 3, 359-379.

Crozier, R.H., Dix, M.W., 1979. Analysis of two genetic models for the innate components of colony odor in social Hymenoptera. Behav. Ecol. Sociobiol. 4, 217-224.

Crozier, R.H., Pamilo, P., 1996. Evolution of Social Insect Colonies, Sex Allocation and Kin Selection. Oxford University Press, Oxford.

D’Ettorre, P., Wenseleers, T., Dawson, J., Hutchinson, S., Boswell, T., Ratnieks, F.L.W., 2006. Wax combs mediate nestmate recognition by guard honeybees. Anim. Behav. 71, 773-779. doi:10.1016/j.anbehav.2005.05.014.

Hamilton, W.D., 1964. The genetical evolution of social behaviour. I \& II. J. Theor. Biol. 7, 1-52.

Hölldobler, B., 1983. Territorial behavior in the green tree ant Oecophylla smaragdina. Biotropica 15, 241-250.

Hornung, V., Latz, E., 2010. Intracellular DNA recognition. Nat. Rev. Immunol. 10, 123-130. doi:10.1038/nri2690.

Katzerke, A., Neumann, P., Pirk, C.W.W., Bliss, P., Moritz, R.F.A., 2006. Seasonal nestmate recognition in the ant Formica exsecta. Behav. Ecol. Sociobiol. 61, 143-150.

Martin, S.J., Helanterä, H., Kiss, K., Lee, Y.R., Drijfhout, F.P., 2009. Polygyny reduces rather than increases nestmate discrimination cue diversity in Formica exsecto ants. Insect. Soc. 56, 375-383. doi:10.1007/s00040-009-0035-z.

Mateo, J.M., 2004. Recognition systems and biological organization: The perception component of social recognition. Ann. Zool. Fenn. 41, 729-745.

Newey, P.S., Robson, S.K.A., Crozier, R.H., 2009. Temporal variation in recognition cues: implications for the social life of weaver ants Oecophylla smaragdina. Anim Behav. 77, 481-488. doi:10.1016/j.anbehav.2008.11.003.

Newey, P.S., Robson, S.K.A., Crozier, R.H., 2010. Know thine enemy: why some weaver ants do but others do not. Behav. Ecol. 21, 381-386. doi:10.1093/beheco/arp201.

Pirk, C.W.W., Neumann, P., Moritz, R.F.A., Pamilo, P., 2001. Intranest relatedness and nestmate recognition in the meadow ant Formica pratensis (R.). Behav. Ecol. Sociobiol. 49, 366-374. doi:10.1007/s002650000315.

Pusey, A., Wolf, M., 1996. Inbreeding avoidance in animals. Trends Ecol. Evol. 11 201-206.

Reeve, H.K., 1989. The evolution of conspecific acceptance thresholds. Am. Nat. 133, 407-435.

Roulston, T.H., Buczkowski, G., Silverman, J., 2003. Nestmate discrimination in ants: effect of bioassay on aggressive behavior. Insect. Soc. 50, 151-159.

Starks, P.T., Watson, R.E., Dipaola, M.J., Dipaola, C.P., 1998. The effect of queen number on nestmate discrimination in the facultatively polygynous ant Pseudomyrmex pallidus (Hymenoptera : Formicidae). Ethology 104, 573-584.

Van Wilgenburg, E., Clémencet, J., Tsutsui, N.D., 2010. Experience influences aggressive behaviour in the Argentine ant. Biol. Lett. 6, 152-155.

van Zweden, J.S., Dreier, S., d'Ettorre, P., 2009. Disentangling environmental and heritable nestmate recognition cues in a carpenter ant. J. Insect Physiol. 55, 158-163, 10.1016/j.jinsphys.2008.11.001.

Vander Meer, R.K., Saliwanchik, D., Lavine, B., 1989. Temporal changes in colony cuticular hydrocarbon patterns of Solenopsis invicta implications for nestmate recognition. J. Chem. Ecol. 15, 2115-2126. 